\title{
Benchmarking Insulin Treatment Persistence Among Patients with Type 2 Diabetes Across Different U.S. Payer Segments
}

\author{
Wenhui Wei, PhD, MS, MBA; Jenny Jiang, MS; Youbei Lou, PhD, MS; \\ Sohini Ganguli, PharmD; and Mark S. Matusik, PharmD
}

\begin{abstract}
BACKGROUND: Treatment persistence with basal insulins is crucial to achieving sustained glycemic control, which is associated with a reduced risk of microvascular disease and other complications of type 2 diabetes (T2D). However, studies suggest that persistence with basal insulin treatment is often poor.

OBJECTIVE: To measure and benchmark real-world basal insulin treatment persistence among patients with T2D across different payer segments in the United States.

METHODS: This was a retrospective observational study of data from a national pharmacy database (Walgreen Co., Deerfield, IL). The analysis included patients with $\mathrm{T} 2 \mathrm{D}$ aged $\geq 18$ years who filled $\geq 1$ prescription for basal insulins between January 2013 and June 2013 (the index prescription) and who had also filled prescriptions for $\geq 1$ oral antidiabetes drug in the database. Patients with claims for premixed insulin were excluded. Treatment persistence was defined as remaining on the study medication(s) during the 1-year follow-up period. Patients were stratified according to treatment history (existing basal insulin users vs. new insulin users), payer segments (commercially insured, Medicare, Medicaid, or cash-pay), type of basal insulin (insulin glargine, insulin detemir, or neutral protamine Hagedorn insulin [NPH]), and device for insulin administration (pen or vial/syringe).
\end{abstract}

RESULTS: A total of 274,102 patients were included in this analysis, $82 \%$ of whom were existing insulin users. In terms of payer segments, $45.3 \%$ of patients were commercially insured, $47.8 \%$ had Medicare, $5.9 \%$ had Medicaid, and $1.1 \%$ were cash-pay. At the 1-year follow-up, basal insulin treatment persistence rate was $66.8 \%$ overall, $61.7 \%$ for new users, and $67.9 \%$ for existing users. In general, for both existing and new basal insulin users, higher persistence rate and duration were associated with Medicare versus cash-pay patients, use of insulin pens versus vial/syringe, and use of insulin glargine versus NPH.

CONCLUSIONS: This large-scale study provides a benchmark of basal insulin treatment persistence across different payers in the United States. Findings indicate that basal insulin persistence patterns are significantly different across different payers, basal insulin types, and devices. This information may be useful in developing targeted approaches to improve T2D patients' persistence with insulin treatment for better glycemic control.

J Manag Care Spec Pharm. 2017;23(3):278-90

Copyright $\odot 2017$, Academy of Managed Care Pharmacy. All rights reserved.

\section{What is already known about this subject}

Persistence with treatment using basal insulins is crucial to achieving sustained glycemic control, which is associated with a reduced risk of microvascular disease and other complications of type 2 diabetes (T2D).

In real-world settings, greater insulin treatment persistence has been linked to improved clinical outcomes and reduced health care utilization.

\section{What this study adds}

This study of a large database of patients with T2D provides a benchmark of basal insulin treatment persistence among patients with T2D across different payer segments in the United States. Knowledge of the association between various payer populations and treatment-related factors on persistence with basal insulin treatment may be useful in helping to design strategies for improving treatment adherence, and therefore glycemic control, in patients with $\mathrm{T} 2 \mathrm{D}$.

T he link between glycemia and the risk of microvascular and macrovascular complications in type 2 diabetes (T2D) is well established. ${ }^{1}$ The goal of treatment is therefore to lower and maintain blood glucose levels, as measured by glycated hemoglobin Alc (Alc), to an individualized target level (usually $<7.0 \%$ ), which is associated with a reduced incidence of such complications. For a high proportion of patients with T2D, due to the diminution of beta cell dysfunction, maintaining this glycemic control will eventually require treatment with insulin as the disease progresses. ${ }^{1}$ Insulin therapy is generally initiated using a long-acting basal insulin analogue, such as insulin glargine, insulin detemir, or regular insulin (e.g., neutral protamine Hagedorn $[\mathrm{NPH}])^{2}{ }^{2}$ Clinical studies have shown that NPH and the long-acting basal insulin analogues have similar efficacy in terms of improving glycemic control; however, the basal analogue insulins have the added advantage of reduced risk of hypoglycemia, which can possibly facilitate more aggressive insulin treatment to an Alc target. ${ }^{3,4}$ However, there is considerable resistance to the initiation of insulin treatment, ${ }^{5}$ which may also contribute to problems with adherence and persistence. Adherence (defined as the extent to which a patient acts in accordance with the 
prescribed interval and dose of and dosing regimen) and persistence (defined as the accumulation of time from initiation to discontinuation of therapy) ${ }^{6}$ to insulin are both factors involved in optimizing outcomes. Treatment adherence with basal insulins is important to achieving sustained glycemic control and, thus, to reduce the risk of microvascular disease and other diabetes-related complications. ${ }^{7}$ However, studies have shown that insulin persistence is suboptimal in patients with $\mathrm{T} 2 \mathrm{D},{ }^{8-10}$ despite persistence having been associated with better clinical outcomes., ${ }^{911}$ A wide range of patient- and treatment-related factors has been shown to affect adherence and persistence to treatment in patients with diabetes, ${ }^{12,13}$ including the financial burden placed on the patient using insulin ${ }^{12}$ such as insurance types. The U.S. health care system is unique among industrialized countries in that it includes commercial (private) health insurance, government health insurance (Medicare), social health insurance (Medicaid), and cash-pay patients. ${ }^{14}$ Given that cost is a major factor affecting access to health care, ${ }^{14}$ health insurance status is likely to influence access to and persistence with T2D medications. While several studies have examined insulin treatment persistence and associated outcomes, ${ }^{9,15}$ there is a lack of real-world data on insulin treatment persistence in various patient populations across different payer segments in the United States. The objective of this analysis was therefore to measure and benchmark real-world basal insulin treatment persistence among patients with T2D in a large national pharmacy database across different payer segments in the United States.

\section{Methods}

\section{Study Design and Patients}

This was a retrospective observational study of data from a large national pharmacy database from Walgreen Co. (Deerfield, IL). Walgreens is one of the nation's largest drugstore chains with more than 8 million customers daily in communities across America. Walgreens operates 8,173 drugstores with a presence in all 50 states, the District of Columbia, Puerto Rico, and the U.S. Virgin Islands and manages one of the largest and most comprehensive prescription data warehouses.

Patients aged $\geq 18$ years who filled $\geq 1$ prescription for the basal insulins glargine, detemir, or NPH between January 2013, and June 2013 (the index basal insulin prescription), were eligible for inclusion in the analysis. Patients with claims for pre-mixed insulin or on 2 different basal insulins on the index date (date of first index basal insulin prescription) and TRICARE patients were excluded. To exclude type 1 diabetes patients, patients were also required to have filled prescriptions for $\geq 1$ oral antidiabetes drug (OAD) in the database. To ensure consistency in data capturing for the baseline and follow-up periods, patients were also required to have filled $\geq 1$ prescription in each of the 4 quarters before (baseline) and each of the 4 quarters after (follow-up). Patients were classified as either existing insulin users ( $\geq 1$ filled prescription for basal insulin in the baseline period) or new insulin users (no claims for any basal insulin during the baseline period).

\section{Outcomes Measures}

Treatment persistence was defined as remaining on the study medication(s) during the 1-year follow-up period. Because patients may switch their basal insulin during the follow-up period, the following 2 types of persistence were considered, depending on the study objectives:

1. When examining differences between payer types, overall basal insulin persistence was studied; therefore, patients might switch to another basal insulin during the follow-up but were still considered persistent with the basal insulin treatment as long as they filled prescriptions for the alternative basal insulin within the expected medication coverage period of their previous basal insulin prescriptions. The same type of persistence was studied when examining differences between pen and vial/syringe as the insulin delivery system.

2. When examining the difference between different index basal insulins, the index basal-specific persistence studied in patients were not considered persistent if they switched to another basal insulin, different from their index basal insulin, during the follow-up.

Due to the fact that basal insulin treatment is non-fixeddosed, the days supply recorded (typically 30 days) in the pharmacy claim is not reliable to estimate actual insulin usage. Therefore, to estimate the expected time of medication coverage of a filled basal insulin prescription, an empirical approach ${ }^{9}$ was used by first creating a look-up table based on patients' pharmacy prescription claims. The look-up table list the 90th percentile of the time interval between the first and second fills among patients with $\geq 1$ refill of their basal insulin within a year. The table was stratified by the specific basal insulin type (glargine, detemir, or NPH) and the metric quantity dispensed as recorded in the first fill (e.g., $10 \mathrm{~mL}, 15 \mathrm{~mL}$ ). This table basically showed the estimated time for the majority of the patients, in this case $90 \%$, to refill their basal insulin prescription, given their prior basal insulin prescription at specific insulin type and dispensed quantity. Study medication was considered to have been discontinued if the prescription was not refilled within the expected time of medication coverage.

The number of treatment-persistent days was defined as the number of days on treatment without discontinuation. Patients who restarted filling a prescription for their index basal insulin after a period of nonpersistence during follow-up were considered to be nonpersistent patients. The proportion of persistent patients (percentage of patients persistent throughout the 1-year follow-up period) and mean duration of persistence (days) were calculated. 


\begin{tabular}{|c|c|c|c|c|c|c|c|c|c|c|c|c|}
\hline \multirow[b]{3}{*}{ Characteristic } & \multicolumn{9}{|c|}{ Existing Users $(\mathrm{N}=224,318)$} & \multirow{2}{*}{\multicolumn{3}{|c|}{$\begin{array}{l}\text { Cash-pay } \\
(n=2,100)\end{array}$}} \\
\hline & \multicolumn{3}{|c|}{$\begin{array}{c}\text { Commercial } \\
(\mathrm{n}=101,063)\end{array}$} & \multicolumn{3}{|c|}{$\begin{array}{c}\text { Medicare } \\
(\mathrm{n}=107,828)\end{array}$} & \multicolumn{3}{|c|}{$\begin{array}{c}\text { Medicaid } \\
(n=13,327)\end{array}$} & & & \\
\hline & 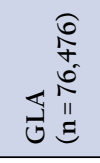 & 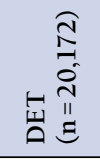 & 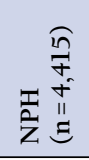 & 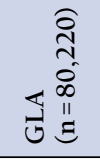 & 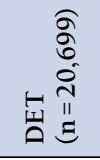 & 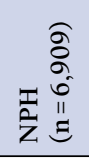 & ङ & 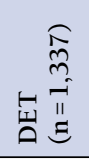 & 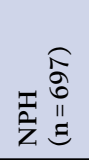 & ङ & 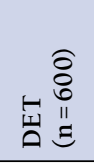 & $=\frac{\sigma}{\stackrel{\sigma}{0}}$ \\
\hline Age in years, mean & 56.4 & 56.0 & 56.1 & 67.9 & 67.7 & 69.6 & 50.5 & 49.6 & 48.4 & 58.3 & 58.5 & 57.3 \\
\hline Females, n (\%) & $\begin{array}{c}36,479 \\
(47.7)\end{array}$ & $\begin{array}{l}9,763 \\
(48.4) \\
\end{array}$ & $\begin{array}{l}2,494 \\
(56.5) \\
\end{array}$ & $\begin{array}{c}46,207 \\
(57.6) \\
\end{array}$ & $\begin{array}{l}12,026 \\
(58.1)\end{array}$ & $\begin{array}{l}4,187 \\
(60.6) \\
\end{array}$ & $\begin{array}{l}7,634 \\
(67.6) \\
\end{array}$ & $\begin{array}{c}947 \\
(70.8) \\
\end{array}$ & $\begin{array}{c}530 \\
(76.0) \\
\end{array}$ & $\begin{array}{c}504 \\
(41.0) \\
\end{array}$ & $\begin{array}{c}245 \\
(40.8) \\
\end{array}$ & $\begin{array}{c}132 \\
(49.1) \\
\end{array}$ \\
\hline \multicolumn{13}{|l|}{ Geographic region, $n(\%)$} \\
\hline Northeast & $\begin{array}{l}7,091 \\
(9.7) \\
\end{array}$ & $\begin{array}{l}2,406 \\
(12.4) \\
\end{array}$ & $\begin{array}{c}460 \\
(11.4) \\
\end{array}$ & $\begin{array}{l}7,879 \\
(10.2) \\
\end{array}$ & $\begin{array}{l}1,908 \\
(9.4) \\
\end{array}$ & $\begin{array}{c}663 \\
(11.2) \\
\end{array}$ & $\begin{array}{l}1,328 \\
(12.0) \\
\end{array}$ & $\begin{array}{c}181 \\
(13.7)\end{array}$ & $\begin{array}{c}88 \\
(12.8) \\
\end{array}$ & $\begin{array}{c}116 \\
(9.6) \\
\end{array}$ & $\begin{array}{c}54 \\
(9.1) \\
\end{array}$ & $\begin{array}{c}29 \\
(11.0) \\
\end{array}$ \\
\hline Midwest & $\begin{array}{c}25,200 \\
(34.4)\end{array}$ & $\begin{array}{l}5,096 \\
(26.2) \\
\end{array}$ & $\begin{array}{l}1,070 \\
(26.6) \\
\end{array}$ & $\begin{array}{l}22,110 \\
(28.7)\end{array}$ & $\begin{array}{l}5,010 \\
(24.7) \\
\end{array}$ & $\begin{array}{l}1,350 \\
(22.9) \\
\end{array}$ & $\begin{array}{l}5,579 \\
(50.5) \\
\end{array}$ & $\begin{array}{c}331 \\
(25.1)\end{array}$ & $\begin{array}{c}281 \\
(40.9) \\
\end{array}$ & $\begin{array}{c}339 \\
(28.0) \\
\end{array}$ & $\begin{array}{c}168 \\
(28.2)\end{array}$ & $\begin{array}{c}58 \\
(22.0) \\
\end{array}$ \\
\hline South & $\begin{array}{l}25,526 \\
(34.9) \\
\end{array}$ & $\begin{array}{l}8,757 \\
(45.0) \\
\end{array}$ & $\begin{array}{l}1,322 \\
(32.9) \\
\end{array}$ & $\begin{array}{l}31,613 \\
(41.1)\end{array}$ & $\begin{array}{l}10,307 \\
(50.8)\end{array}$ & $\begin{array}{l}2,648 \\
(44.9) \\
\end{array}$ & $\begin{array}{l}2,985 \\
(27.0) \\
\end{array}$ & $\begin{array}{c}643 \\
(48.8) \\
\end{array}$ & $\begin{array}{c}188 \\
(27.4) \\
\end{array}$ & $\begin{array}{c}478 \\
(39.5) \\
\end{array}$ & $\begin{array}{c}271 \\
(45.6) \\
\end{array}$ & $\begin{array}{c}107 \\
(40.5) \\
\end{array}$ \\
\hline West & $\begin{array}{l}15,351 \\
(21.0)\end{array}$ & $\begin{array}{l}3,204 \\
(16.5) \\
\end{array}$ & $\begin{array}{l}1,170 \\
(29.1) \\
\end{array}$ & $\begin{array}{l}15,332 \\
(19.9)\end{array}$ & $\begin{array}{l}3,047 \\
(15.0)\end{array}$ & $\begin{array}{l}1,241 \\
(21.0) \\
\end{array}$ & $\begin{array}{l}1,156 \\
(10.5) \\
\end{array}$ & $\begin{array}{c}163 \\
(12.4) \\
\end{array}$ & $\begin{array}{c}130 \\
(18.9) \\
\end{array}$ & $\begin{array}{c}278 \\
(23.0) \\
\end{array}$ & $\begin{array}{c}102 \\
(17.1)\end{array}$ & $\begin{array}{c}70 \\
(26.5) \\
\end{array}$ \\
\hline \multicolumn{13}{|l|}{ OAD, n (\%) } \\
\hline Metformin & $\begin{array}{l}43,196 \\
(56.5) \\
\end{array}$ & $\begin{array}{c}11,538 \\
(57.2)\end{array}$ & $\begin{array}{l}2,270 \\
(51.4)\end{array}$ & $\begin{array}{l}35,831 \\
(44.7) \\
\end{array}$ & $\begin{array}{l}9,469 \\
(45.8) \\
\end{array}$ & $\begin{array}{l}2,911 \\
(42.1)\end{array}$ & $\begin{array}{l}6,316 \\
(55.9) \\
\end{array}$ & $\begin{array}{l}719 \\
(53.8) \\
\end{array}$ & $\begin{array}{c}345 \\
(49.5) \\
\end{array}$ & $\begin{array}{l}775 \\
(63.0) \\
\end{array}$ & $\begin{array}{c}363 \\
(60.5) \\
\end{array}$ & $\begin{array}{c}160 \\
(59.5) \\
\end{array}$ \\
\hline DPP-4 inhibitors & $\begin{array}{c}11,809 \\
(15.4)\end{array}$ & $\begin{array}{l}3,960 \\
(19.6) \\
\end{array}$ & $\begin{array}{l}341 \\
(7.7)\end{array}$ & $\begin{array}{l}10,303 \\
(12.8) \\
\end{array}$ & $\begin{array}{l}3,235 \\
(15.6) \\
\end{array}$ & $\begin{array}{l}367 \\
(5.3) \\
\end{array}$ & $\begin{array}{l}1,340 \\
(11.9) \\
\end{array}$ & $\begin{array}{c}203 \\
(15.2) \\
\end{array}$ & $\begin{array}{c}34 \\
(4.9) \\
\end{array}$ & $\begin{array}{c}80 \\
(6.5) \\
\end{array}$ & $\begin{array}{c}48 \\
(8.0)\end{array}$ & $\begin{array}{c}6 \\
(2.2) \\
\end{array}$ \\
\hline Sulfonylureas & $\begin{array}{c}21,276 \\
(27.8) \\
\end{array}$ & $\begin{array}{r}5,746 \\
(28.5) \\
\end{array}$ & $\begin{array}{l}1,045 \\
(23.7) \\
\end{array}$ & $\begin{array}{c}23,614 \\
(29.4) \\
\end{array}$ & $\begin{array}{l}6,515 \\
(31.5) \\
\end{array}$ & $\begin{array}{l}1,725 \\
(25.0) \\
\end{array}$ & $\begin{array}{r}2,833 \\
(25.1) \\
\end{array}$ & $\begin{array}{c}344 \\
(25.7) \\
\end{array}$ & $\begin{array}{c}136 \\
(19.5) \\
\end{array}$ & $\begin{array}{c}435 \\
(35.3) \\
\end{array}$ & $\begin{array}{c}210 \\
(35.0) \\
\end{array}$ & $\begin{array}{c}93 \\
(34.6) \\
\end{array}$ \\
\hline Thiazolidinediones & $\begin{array}{c}2,322 \\
(3.0)\end{array}$ & $\begin{array}{l}667 \\
(3.3) \\
\end{array}$ & $\begin{array}{c}111 \\
(2.5)\end{array}$ & $\begin{array}{c}1,508 \\
(1.9)\end{array}$ & $\begin{array}{l}386 \\
(1.9) \\
\end{array}$ & $\begin{array}{c}105 \\
(1.5)\end{array}$ & $\begin{array}{c}477 \\
(4.2) \\
\end{array}$ & $\begin{array}{c}59 \\
(4.4) \\
\end{array}$ & $\begin{array}{c}25 \\
(3.6) \\
\end{array}$ & $\begin{array}{c}13 \\
(1.1)\end{array}$ & $\begin{array}{c}10 \\
(1.7) \\
\end{array}$ & $\begin{array}{c}1 \\
(0.4) \\
\end{array}$ \\
\hline a-glucosidase inhibitors & $\begin{array}{l}273 \\
(0.4) \\
\end{array}$ & $\begin{array}{c}87 \\
(0.4) \\
\end{array}$ & $\begin{array}{c}25 \\
(0.6) \\
\end{array}$ & $\begin{array}{c}401 \\
(0.5) \\
\end{array}$ & $\begin{array}{c}116 \\
(0.6) \\
\end{array}$ & $\begin{array}{c}42 \\
(0.6) \\
\end{array}$ & $\begin{array}{c}43 \\
(0.4) \\
\end{array}$ & $\begin{array}{c}3 \\
(0.2) \\
\end{array}$ & $\begin{array}{c}1 \\
(0.1)\end{array}$ & $\begin{array}{c}0 \\
(0.0)\end{array}$ & $\begin{array}{c}5 \\
(0.8) \\
\end{array}$ & $\begin{array}{c}2 \\
(0.7)\end{array}$ \\
\hline Number of OADs, mean (SD) & $\begin{array}{c}1.2 \\
(1.1)\end{array}$ & $\begin{array}{c}1.2 \\
(1.1) \\
\end{array}$ & $\begin{array}{c}1.0 \\
(1.1)\end{array}$ & $\begin{array}{c}1.0 \\
(1.1) \\
\end{array}$ & $\begin{array}{c}1.1 \\
(1.1)\end{array}$ & $\begin{array}{c}0.9 \\
(1.0)\end{array}$ & $\begin{array}{c}1.2 \\
(1.2)\end{array}$ & $\begin{array}{c}1.2 \\
(1.2)\end{array}$ & $\begin{array}{c}1.0 \\
(1.1)\end{array}$ & $\begin{array}{c}1.2 \\
(1.1)\end{array}$ & $\begin{array}{c}1.2 \\
(1.1) \\
\end{array}$ & $\begin{array}{c}1.2 \\
(1.0) \\
\end{array}$ \\
\hline GLP-1 RA, n (\%) & $\begin{array}{c}6,935 \\
(9.1) \\
\end{array}$ & $\begin{array}{l}2,616 \\
(13.0)\end{array}$ & $\begin{array}{c}244 \\
(5.5) \\
\end{array}$ & $\begin{array}{c}3,236 \\
(4.0)\end{array}$ & $\begin{array}{l}1,287 \\
(6.2)\end{array}$ & $\begin{array}{c}172 \\
(2.5)\end{array}$ & $\begin{array}{c}433 \\
(3.8) \\
\end{array}$ & $\begin{array}{c}103 \\
(7.7)\end{array}$ & $\begin{array}{c}13 \\
(1.9)\end{array}$ & $\begin{array}{c}35 \\
(2.8) \\
\end{array}$ & $\begin{array}{c}21 \\
(3.5) \\
\end{array}$ & $\begin{array}{c}2 \\
(0.7)\end{array}$ \\
\hline Rapid-acting/regular insulin, n (\%) & $\begin{array}{c}32,854 \\
(43.0) \\
\end{array}$ & $\begin{array}{l}8,951 \\
(44.4) \\
\end{array}$ & $\begin{array}{l}2,288 \\
(51.8) \\
\end{array}$ & $\begin{array}{c}33,299 \\
(41.5) \\
\end{array}$ & $\begin{array}{l}8,900 \\
(43.0) \\
\end{array}$ & $\begin{array}{l}2,557 \\
(37.0) \\
\end{array}$ & $\begin{array}{l}5,937 \\
(52.3) \\
\end{array}$ & $\begin{array}{c}737 \\
(55.1) \\
\end{array}$ & $\begin{array}{c}422 \\
(60.6) \\
\end{array}$ & $\begin{array}{c}308 \\
(25.0) \\
\end{array}$ & $\begin{array}{c}174 \\
(29.0) \\
\end{array}$ & $\begin{array}{c}67 \\
(24.9) \\
\end{array}$ \\
\hline
\end{tabular}

\section{Statistical Analyses}

The primary independent variable in this analysis was payer type (commercially insured, Medicare, Medicaid, or cash-pay); analyses were also performed for insulin type (glargine, detemir, or NPH), device (pen or vial/syringe), and patient cohorts (existing or new users). Descriptive data using an intent-to-treat analysis are reported. Findings were stratified by existing basal insulin users and new insulin users. Statistical comparisons between the covariates were performed using Student's t-tests or chi-square tests, as appropriate. Time to treatment discontinuation was evaluated using Kaplan-Meier survival curves and comparisons between cohorts were made using log-rank tests.

Multivariate analyses were performed using logistic regression models to estimate the relationship between overall basal insulin treatment persistence, index insulin type, payer type, and device type, adjusting for age; gender; region; baseline
OAD usage; baseline rapid-acting/regular insulin usage; baseline antihypertension and antihyperlipidemia medication usage; and copayment for the index basal insulin for overall patients, existing users, and new users. Cox proportional hazards regression models were also used to identify independent factors associated with patients' time to nonpersistence with their overall basal insulin treatment after adjusting for all the same covariates as in the logistic regression models.

The level of significance for hypothesis testing was set at $P<0.05$. All data analyses were performed with SAS 9.4 software (SAS Institute, Cary, NC).

\section{Results}

\section{Patient Demographic and Clinical Characteristics}

A total of 274,102 patients were included in this analysis. The mean age of patients was 61.3 years, about half (54.1\%) were 
Benchmarking Insulin Treatment Persistence Among Patients with Type 2 Diabetes Across Different U.S. Payer Segments

TABLE 1 Baseline Patient Demographic and Clinical Characteristics for Existing and New Users of Basal Insulin (continued)

\begin{tabular}{|c|c|c|c|c|c|c|c|c|c|c|c|c|}
\hline \multirow[b]{3}{*}{ Characteristic } & \multicolumn{9}{|c|}{ New Users $(\mathrm{N}=49,784)$} & \multirow{2}{*}{\multicolumn{3}{|c|}{$\begin{array}{c}\text { Cash-pay } \\
(n=662)\end{array}$}} \\
\hline & \multicolumn{3}{|c|}{$\begin{array}{c}\text { Commercial } \\
(\mathrm{n}=23,091)\end{array}$} & \multicolumn{3}{|c|}{$\begin{array}{c}\text { Medicare } \\
(\mathrm{n}=23,185)\end{array}$} & \multicolumn{3}{|c|}{$\begin{array}{c}\text { Medicaid } \\
(\mathrm{n}=2,846)\end{array}$} & & & \\
\hline & ङ & 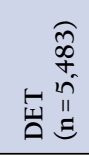 & 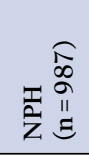 & ङ & 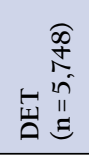 & $\begin{array}{r}\widehat{\sigma} \\
\overline{0} \\
\bar{z} \\
\bar{z}\end{array}$ & 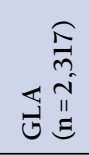 & 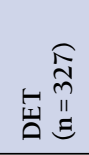 & 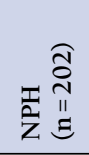 & 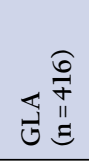 & 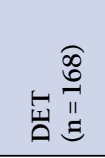 & 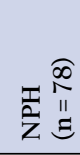 \\
\hline Age in years, mean & 54.9 & 54.4 & 47.9 & 67.2 & 66.9 & 67.1 & 48.5 & 47.5 & 40.2 & 57.7 & 60.1 & 52.3 \\
\hline Female, n (\%) & $\begin{array}{l}8,244 \\
(49.6) \\
\end{array}$ & $\begin{array}{l}2,741 \\
(50.0) \\
\end{array}$ & $\begin{array}{c}694 \\
(70.3) \\
\end{array}$ & $\begin{array}{l}9,392 \\
(57.0) \\
\end{array}$ & $\begin{array}{l}3,294 \\
(57.3) \\
\end{array}$ & $\begin{array}{c}407 \\
(58.9) \\
\end{array}$ & $\begin{array}{l}1,587 \\
(68.5) \\
\end{array}$ & $\begin{array}{c}234 \\
(71.6) \\
\end{array}$ & $\begin{array}{c}170 \\
(84.2)\end{array}$ & $\begin{array}{c}178 \\
(42.8) \\
\end{array}$ & $\begin{array}{c}65 \\
(38.7) \\
\end{array}$ & $\begin{array}{c}40 \\
(51.3) \\
\end{array}$ \\
\hline \multicolumn{13}{|l|}{ Geographic region, $n$ (\%) } \\
\hline Northeast & $\begin{array}{l}1,636 \\
(10.3)\end{array}$ & $\begin{array}{c}636 \\
(12.1) \\
\end{array}$ & $\begin{array}{c}107 \\
(11.6)\end{array}$ & $\begin{array}{l}1,583 \\
(10.1) \\
\end{array}$ & $\begin{array}{l}515 \\
(9.2) \\
\end{array}$ & $\begin{array}{c}86 \\
(10.1) \\
\end{array}$ & $\begin{array}{c}244 \\
(10.8) \\
\end{array}$ & $\begin{array}{c}40 \\
(12.2)\end{array}$ & $\begin{array}{c}20 \\
(10.1)\end{array}$ & $\begin{array}{c}48 \\
(11.7)\end{array}$ & $\begin{array}{c}20 \\
(12.0)\end{array}$ & $\begin{array}{c}2 \\
(2.7)\end{array}$ \\
\hline Midwest & $\begin{array}{l}5,151 \\
(32.5) \\
\end{array}$ & $\begin{array}{l}1,245 \\
(23.6) \\
\end{array}$ & $\begin{array}{c}253 \\
(27.4) \\
\end{array}$ & $\begin{array}{l}4,331 \\
(27.5) \\
\end{array}$ & $\begin{array}{l}1,237 \\
(22.0) \\
\end{array}$ & $\begin{array}{c}168 \\
(19.7) \\
\end{array}$ & $\begin{array}{l}1,084 \\
(48.0) \\
\end{array}$ & $\begin{array}{c}85 \\
(26.0) \\
\end{array}$ & $\begin{array}{c}91 \\
(46.0) \\
\end{array}$ & $\begin{array}{c}108 \\
(26.2) \\
\end{array}$ & $\begin{array}{c}43 \\
(25.8) \\
\end{array}$ & $\begin{array}{c}23 \\
(31.1) \\
\end{array}$ \\
\hline South & $\begin{array}{l}5,965 \\
(37.6)\end{array}$ & $\begin{array}{l}2,590 \\
(49.1)\end{array}$ & $\begin{array}{c}302 \\
(32.7)\end{array}$ & $\begin{array}{l}6,771 \\
(43.0) \\
\end{array}$ & $\begin{array}{l}3,132 \\
(55.6)\end{array}$ & $\begin{array}{c}417 \\
(49.0) \\
\end{array}$ & $\begin{array}{c}685 \\
(30.3) \\
\end{array}$ & $\begin{array}{c}167 \\
(51.1)\end{array}$ & $\begin{array}{c}46 \\
(23.2) \\
\end{array}$ & $\begin{array}{c}167 \\
(40.5)\end{array}$ & $\begin{array}{c}83 \\
(49.7) \\
\end{array}$ & $\begin{array}{c}34 \\
(45.9) \\
\end{array}$ \\
\hline West & $\begin{array}{l}3,123 \\
(19.7) \\
\end{array}$ & $\begin{array}{c}804 \\
(15.2)\end{array}$ & $\begin{array}{c}261 \\
(28.3)\end{array}$ & $\begin{array}{l}3,058 \\
(19.4) \\
\end{array}$ & $\begin{array}{c}745 \\
(13.2) \\
\end{array}$ & $\begin{array}{c}180 \\
(21.2)\end{array}$ & $\begin{array}{c}246 \\
(10.9)\end{array}$ & $\begin{array}{c}35 \\
(10.7)\end{array}$ & $\begin{array}{c}41 \\
(20.7)\end{array}$ & $\begin{array}{c}89 \\
(21.6) \\
\end{array}$ & $\begin{array}{c}21 \\
(12.6)\end{array}$ & $\begin{array}{c}15 \\
(20.3) \\
\end{array}$ \\
\hline \multicolumn{13}{|l|}{$\mathrm{OAD}, \mathbf{n}(\%)$} \\
\hline Metformin & $\begin{array}{l}9,860 \\
(59.3)\end{array}$ & $\begin{array}{l}3,292 \\
(60.0)\end{array}$ & $\begin{array}{c}553 \\
(56.0) \\
\end{array}$ & $\begin{array}{l}7,718 \\
(46.8) \\
\end{array}$ & $\begin{array}{l}2,741 \\
(47.7) \\
\end{array}$ & $\begin{array}{c}395 \\
(41.1)\end{array}$ & $\begin{array}{l}1,397 \\
(60.3)\end{array}$ & $\begin{array}{c}179 \\
(54.7)\end{array}$ & $\begin{array}{c}111 \\
(55.0)\end{array}$ & $\begin{array}{c}241 \\
(57.9)\end{array}$ & $\begin{array}{c}103 \\
(61.3)\end{array}$ & $\begin{array}{c}51 \\
(65.4) \\
\end{array}$ \\
\hline DPP-4 inhibitors & $\begin{array}{l}3,870 \\
(23.3)\end{array}$ & $\begin{array}{l}1,572 \\
(28.7)\end{array}$ & $\begin{array}{c}79 \\
(8.0)\end{array}$ & $\begin{array}{l}3,435 \\
(20.9) \\
\end{array}$ & $\begin{array}{l}1,412 \\
(24.6)\end{array}$ & $\begin{array}{c}94 \\
(9.8)\end{array}$ & $\begin{array}{c}430 \\
(18.6) \\
\end{array}$ & $\begin{array}{c}71 \\
(21.7)\end{array}$ & $\begin{array}{c}20 \\
(9.9)\end{array}$ & $\begin{array}{c}37 \\
(8.9) \\
\end{array}$ & $\begin{array}{c}14 \\
(8.3)\end{array}$ & $\begin{array}{c}7 \\
(9.0)\end{array}$ \\
\hline Sulfonylureas & $\begin{array}{l}7,245 \\
(43.6) \\
\end{array}$ & $\begin{array}{l}2,407 \\
(43.9)\end{array}$ & $\begin{array}{c}352 \\
(35.7)\end{array}$ & $\begin{array}{l}7,510 \\
(45.6) \\
\end{array}$ & $\begin{array}{l}2,728 \\
(47.5) \\
\end{array}$ & $\begin{array}{c}349 \\
(36.3)\end{array}$ & $\begin{array}{c}970 \\
(41.9)\end{array}$ & $\begin{array}{c}132 \\
(40.4)\end{array}$ & $\begin{array}{c}81 \\
(40.1)\end{array}$ & $\begin{array}{c}220 \\
(52.9)\end{array}$ & $\begin{array}{c}98 \\
(58.3) \\
\end{array}$ & $\begin{array}{c}46 \\
(59.0) \\
\end{array}$ \\
\hline Thiazolidinediones & $\begin{array}{l}226 \\
(1.4) \\
\end{array}$ & $\begin{array}{c}81 \\
(1.5) \\
\end{array}$ & $\begin{array}{c}9 \\
(0.9) \\
\end{array}$ & $\begin{array}{l}203 \\
(1.2) \\
\end{array}$ & $\begin{array}{c}78 \\
(1.4) \\
\end{array}$ & $\begin{array}{c}12 \\
(1.3) \\
\end{array}$ & $\begin{array}{c}70 \\
(3.0) \\
\end{array}$ & $\begin{array}{c}6 \\
(1.8) \\
\end{array}$ & $\begin{array}{c}3 \\
(1.5) \\
\end{array}$ & $\begin{array}{c}1 \\
(0.2) \\
\end{array}$ & $\begin{array}{c}0 \\
(0.0) \\
\end{array}$ & $\begin{array}{c}0 \\
(0.0) \\
\end{array}$ \\
\hline a-glucosidase inhibitors & $\begin{array}{c}95 \\
(0.6) \\
\end{array}$ & $\begin{array}{c}39 \\
(0.7) \\
\end{array}$ & $\begin{array}{c}6 \\
(0.6) \\
\end{array}$ & $\begin{array}{c}139 \\
(0.8) \\
\end{array}$ & $\begin{array}{c}45 \\
(0.8) \\
\end{array}$ & $\begin{array}{c}4 \\
(0.4) \\
\end{array}$ & $\begin{array}{c}5 \\
(0.2) \\
\end{array}$ & $\begin{array}{c}2 \\
(0.6) \\
\end{array}$ & $\begin{array}{c}0 \\
(0.0) \\
\end{array}$ & $\begin{array}{c}4 \\
(1.0) \\
\end{array}$ & $\begin{array}{c}4 \\
(2.4) \\
\end{array}$ & $\begin{array}{c}0 \\
(0.0) \\
\end{array}$ \\
\hline Number of OADs, mean (SD) & $\begin{array}{c}1.5 \\
(1.3) \\
\end{array}$ & $\begin{array}{c}1.5 \\
(1.3) \\
\end{array}$ & $\begin{array}{c}1.3 \\
(1.2) \\
\end{array}$ & $\begin{array}{c}1.4 \\
(1.3) \\
\end{array}$ & $\begin{array}{c}1.5 \\
(1.3) \\
\end{array}$ & $\begin{array}{c}1.1 \\
(1.2) \\
\end{array}$ & $\begin{array}{c}1.6 \\
(1.4) \\
\end{array}$ & $\begin{array}{c}1.4 \\
(1.4) \\
\end{array}$ & $\begin{array}{c}1.3 \\
(1.3) \\
\end{array}$ & $\begin{array}{c}1.4 \\
(1.2) \\
\end{array}$ & $\begin{array}{c}1.6 \\
(1.2) \\
\end{array}$ & $\begin{array}{c}1.7 \\
(1.4) \\
\end{array}$ \\
\hline$\overline{\text { GLP-1, n (\%) }}$ & $\begin{array}{l}1,737 \\
(10.5) \\
\end{array}$ & $\begin{array}{c}849 \\
(15.5) \\
\end{array}$ & $\begin{array}{c}49 \\
(5.0) \\
\end{array}$ & $\begin{array}{c}799 \\
(4.8) \\
\end{array}$ & $\begin{array}{l}382 \\
(6.6) \\
\end{array}$ & $\begin{array}{c}23 \\
(2.4) \\
\end{array}$ & $\begin{array}{c}103 \\
(4.5) \\
\end{array}$ & $\begin{array}{c}24 \\
(7.3) \\
\end{array}$ & $\begin{array}{c}3 \\
(1.5) \\
\end{array}$ & $\begin{array}{c}11 \\
(2.64) \\
\end{array}$ & $\begin{array}{c}8 \\
(4.76) \\
\end{array}$ & $\begin{array}{c}0 \\
(0.0) \\
\end{array}$ \\
\hline Rapid-acting/regular insulin, n (\%) & $\begin{array}{l}4,093 \\
(24.6) \\
\end{array}$ & $\begin{array}{l}1,308 \\
(23.9) \\
\end{array}$ & $\begin{array}{c}414 \\
(41.9) \\
\end{array}$ & $\begin{array}{l}4,163 \\
(25.3) \\
\end{array}$ & $\begin{array}{l}1,406 \\
(24.5) \\
\end{array}$ & $\begin{array}{c}256 \\
(26.6) \\
\end{array}$ & $\begin{array}{c}722 \\
(31.2) \\
\end{array}$ & $\begin{array}{c}108 \\
(33.0) \\
\end{array}$ & $\begin{array}{c}110 \\
(54.5) \\
\end{array}$ & $\begin{array}{c}76 \\
(18.3) \\
\end{array}$ & $\begin{array}{c}18 \\
(10.7) \\
\end{array}$ & $\begin{array}{c}18 \\
(23.1) \\
\end{array}$ \\
\hline
\end{tabular}

DET = insulin detemir; DPP-4 = dipeptidyl peptidase-4; GLA = insulin glargine; GLP-1 RA=glucagon-like peptide-1 receptor antagonist; NPH =neutral protamine Hagedorn insulin; $\mathrm{OAD}=$ oral antidiabetes drug.

women, and in terms of payer segments, $45.3 \%$ were commercially insured, $47.8 \%$ were covered by Medicare, $5.9 \%$ were covered by Medicaid, and $1.1 \%$ were cash-pay patients. Substantial demographic variations by payer type were seen, with Medicare patients being the oldest (mean age 56.3 years, 67.9 years, 50.3 years, and 58.2 years, respectively), Medicaid patients being the youngest yet having the highest proportion of females, and cash-pay patients having the lowest proportion of females (48.3\%, 57.9\%, 68.3\%, and $42.0 \%$, respectively). Overall, $82 \%$ of patients were existing basal insulin users. At the index fill, $75 \%$ of all patients received insulin glargine, $20 \%$ received insulin detemir, and 5\% received NPH. Detailed patient demographic and clinical characteristic information are stratified by payer type and basal insulin type and presented in Table 1.

\section{Overall Basal Insulin Treatment Persistence}

Overall, the rate of persistence with basal insulin at the 1-year follow-up was $66.8 \%$ (95\% confidence interval $[\mathrm{CI}]=66.6-67.0)$ and mean duration of persistence was 307.9 days (standard deviation $[\mathrm{SD}]=85.2$. . Compared with new users, existing users had significantly higher persistence rates (67.9\%, 95\% $\mathrm{CI}=67.7-68.1$ vs. $61.6 \%, 95 \% \mathrm{CI}=61.2-62.1 ; P<0.0001)$ but lower duration of persistence (mean \pm SD: $307.5 \pm 87.9$ vs. $309.8 \pm 71.8$ days; $P \leq 0.0001)$.

\section{Basal Insulin Treatment Persistence by Payer}

Overall, persistence with basal insulin at the 1-year follow-up (both rates and duration) was lowest for cash-pay patients (mean rate $=58.6 \%, 95 \%$ CI $=56.7-60.4$; mean duration $=293$ days, $S D=93.3$ ) and highest for Medicare patients (mean 
rate $=68.5 \%, 95 \% \mathrm{CI}=68.2-68.7$; mean duration $=312$ days, $\mathrm{SD}=81.3$; all $\mathrm{P}<0.0001)$

Existing Users. Among existing users, the persistence rate for commercially insured patients $(67.0 \%, 95 \% \mathrm{CI}=66.8-67.3)$ was significantly higher $(P<0.0001)$ than the rate for cash-pay and Medicaid patients $(61.1 \%, 95 \% \mathrm{CI}=59.0-63.2$ and $63.2 \%$, $95 \% \mathrm{CI}=62.4-64.0)$ but was significantly lower $(P<0.0001)$ than the rate for Medicare patients $(69.5 \%, 95 \% \mathrm{CI}=69.2-69.7$; Figure 1).

The mean \pm SD duration of persistence with basal insulin at 1 year was significantly higher for commercially insured patients when compared with cash-pay patients ( $305 \pm 90.9$ vs. $294 \pm 96.8$ days; $P<0.0001)$ and Medicaid patients $(305 \pm 90.9$ vs. $298 \pm 94.5$ days; $P<0.0001)$ but significantly lower when compared with Medicare patients $(305 \pm 90.9$ vs. $312 \pm 83.8$ days; $P<0.0001$; Figure 1). Kaplan-Meier curves showed that Medicare patients were less likely to discontinue earlier than other payer types $(P<0.0001$; Figure 2$)$.

New Users. Among new users, compared with commercially insured patients, the persistence rate was similar for Medicaid patients $(59.5 \%, 95 \% \mathrm{CI}=57.7-61.3$ vs. $60.0 \%, 95 \% \mathrm{CI}=59.3$ 60.6) but significantly higher for Medicare patients (63.9\%, 95\% CI $=63.3-64.5)$ and significantly lower for cash-pay patients (50.6\%, 95\% CI =46.8-54.4; both $P<0.0001$; Figure 1).

Mean \pm SD duration of persistence with basal insulin at 1-year follow-up was similar for commercially insured and Medicaid patients ( $307 \pm 73.8$ days and $305 \pm 77.7$ days, respectively) but significantly higher for commercially insured patients compared with cash-pay patients $(292 \pm 81.4$ days; $P<0.0001)$ and significantly lower compared with Medicare patients ( $314 \pm 68.3$ days; $P<0.0001$; Figure 1). Kaplan-Meier curves showed that Medicare patients were least likely to discontinue earlier than other payer types $(P<0.0001$; Figure 2$)$.

Index Basal Insulin Treatment Persistence by Basal Insulin Type Overall, mean persistence rate and duration with index insulin were $64.6 \%$ (95\% CI $=64.5-64.8)$ and $304 \pm 87.4$ days, respectively. For existing users, the rates were 65.8\% (95\% $\mathrm{CI}=65.6-66.0)$ and $303.5 \pm 90.3$ days, respectively. For new users, they were $59.2 \%(95 \% \mathrm{CI}=58.8-59.6)$ and $306.3 \pm 73.1$ days, respectively.

Existing Users. Among existing users, persistence rate was significantly higher with insulin glargine versus both insulin detemir and NPH in commercially insured (66.5\%, 95\% $\mathrm{CI}=66.2-66.9$ vs. $63.8 \%, 95 \% \mathrm{CI}=63.2-64.5$ and $54.7 \%, 95 \%$ $\mathrm{CI}=53.3-56.2$, respectively; both $\mathrm{P}<0.0001)$ and Medicaid patients $(62.4 \%, 95 \% \mathrm{CI}=61.5-63.3$ vs. $58.0 \%, 95 \% \mathrm{CI}=55.4-$ 60.7 and $43.0 \%, 95 \% \mathrm{CI}=39.4-46.7 ; P=0.0019$ and $P<0.0001$, respectively) and versus NPH only in Medicare patients $(67.4 \%$, 95\% CI=67.1-67.7 vs. $61.4 \%, 95 \% \quad C I=0.3-62.6 ; P<0.0001$; Figure 3). Persistence duration \pm SD was significantly longer for insulin glargine versus both insulin detemir and NPH in commercially insured ( $304 \pm 91.4$ vs. $300 \pm 92.0$ and $278 \pm 104.3$ days; both $P<0.0001)$ and Medicaid patients $(296 \pm 94.8$ vs. $290 \pm 95.7$ and $260 \pm 104.2$ days; both $P<0.0001$ ) and versus NPH only in Medicare patients $(307 \pm 87.2$ vs. $296 \pm 94.5$ days; $P<0.0001$; Figure 3). Kaplan-Meier curves showed that insulin glargine patients were less likely to discontinue earlier than patients on insulin detemir or NPH $(P<0.0001$; data not shown).

New Users. Among new users, persistence rate was significantly higher with insulin glargine versus both insulin detemir and NPH in Medicaid patients $(61.6 \%, 95 \% \mathrm{CI}=59.6-63.5$ vs. $50.2 \%, 95 \% \mathrm{CI}=44.7-55.6$ and $21.3 \%, 95 \% \mathrm{CI}=15.6-26.9$; both $P<0.0001)$ and versus NPH only in commercially insured (58.7\%, 95\% CI=57.9-59.4 vs. $38.0 \%, 95 \% \mathrm{CI}=35.0-41.0$ ), Medicare (61.9\%, 95\% CI=61.1-62.9 vs. $48.3 \%, 95 \% \mathrm{CI}=45.1$ 51.5), and cash-pay patients $(49.3 \%, 95 \% \mathrm{CI}=44.5-54.1$ vs. 33.3\%, 95\% CI=22.9-43.8; all $P<0.0001$; Figure 3). Persistence duration $\pm S D$ was significantly longer for insulin glargine (308 \pm 75.1 days) versus both insulin detemir $(289 \pm 82.7$ days $)$ and NPH $(246 \pm 88.3$ days) in Medicaid patients (both $P<0.0001)$ and versus NPH only in commercially insured ( $304 \pm 75.3$ days vs. $275 \pm 84.9$ days), Medicare ( $311 \pm 70.2$ days vs. $292 \pm 76.1$ days), and cash-pay ( $298 \pm 69.5$ days vs. $222 \pm 116.2$ days) patients (all $P<0.0001$; Figure 3). Kaplan-Meier curves showed that NPH patients were more likely to discontinue earlier than other patients $(P<0.0001$; data not shown).

\section{Basal Insulin Treatment Persistence by Index Insulin Device}

Overall, mean basal insulin persistence rate and duration \pm SD for those who had a pen as their index insulin device were 67.9\% (95\% CI=67.9-68.1) and 311.2 \pm 81.5 days, both of which were significantly higher than those with a vial/syringe as their index insulin device $(65.0 \%, 95 \% \mathrm{CI}=64.8-65.3$ and $302.9 \pm 90.4$ days; both $P<0.0001)$.

Existing Users. Among existing users, the persistence rate for pens overall was $69.0 \%(95 \% \mathrm{CI}=68.7-69.2)$ and for vial/ syringe was $66.4 \%$ (95\% CI=66.1-66.7; Figure 4). The persistence rate was significantly higher for pen versus vial/syringe users for commercially insured $(67.8 \%, 95 \% \mathrm{CI}=67.4-68.2$ vs. 65.6\%, 95\% CI $65.1-66.1)$, Medicare $(70.8 \%, 95 \% \mathrm{CI}=70.5$ 71.2 vs. $67.8 \%, 95 \% \mathrm{CI}=67.3-68.2)$, and cash-pay patients (66.7\%, 95\% CI =63.5-69.9 vs. $57.3 \%, 95 \% C I=54.6-60.0$; all $P<0.0001)$. There was no significant difference in the persistence rate for the 2 devices for Medicaid patients (63.5\%, $95 \% \mathrm{CI}=62.2-64.7$ vs. $63.0 \%, 95 \% \mathrm{CI}=61.9-64.0 ; P=0.5485)$. Persistence duration \pm SD for pen users ranged from $301 \pm 89.6$ to $315 \pm 79.4$ days and for vial/syringe users ranged from $285 \pm 102.0$ to $307 \pm 88.7$ days (Figure 4). Pen use was associated with significantly longer duration of persistence compared with vial/syringe use for all payer types (all $P<0.001$ ). Kaplan-Meier curves of time to discontinuation showed that 


\section{FIGURE 1 Persistence by Payer}
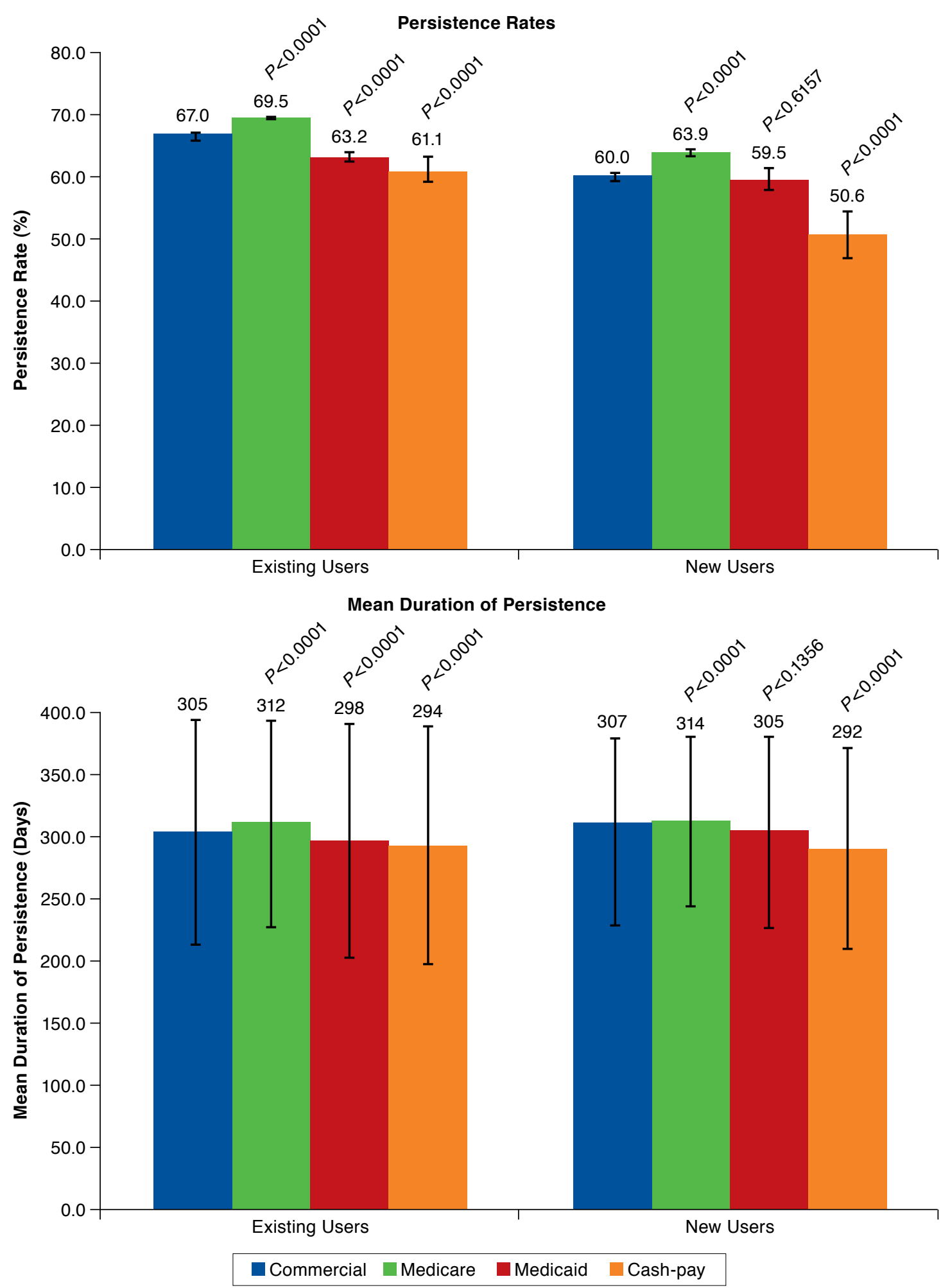

Note: Error bars represent 95\% confidence intervals (top) or standard deviations (bottom). P values compared with commercially insured patients. 
Existing Users

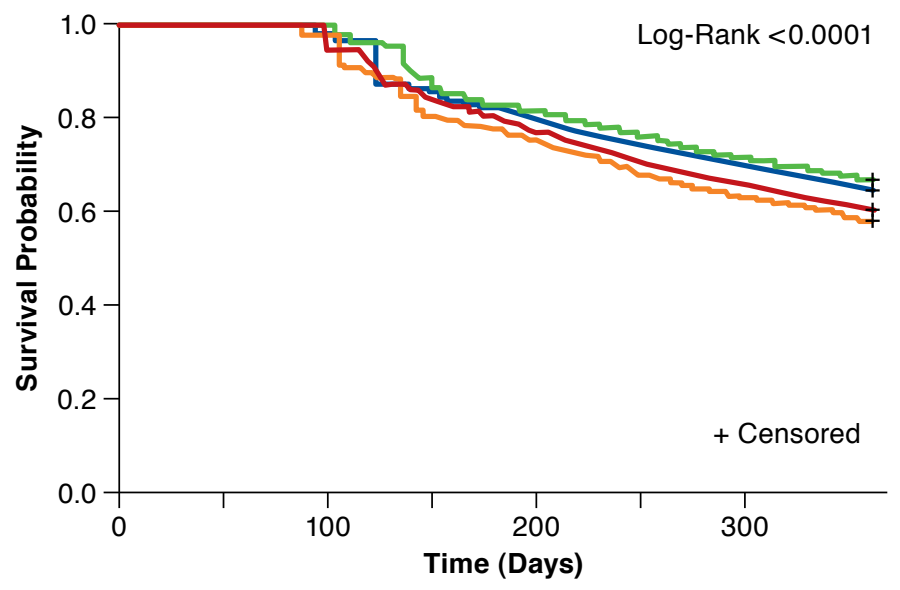

New Users

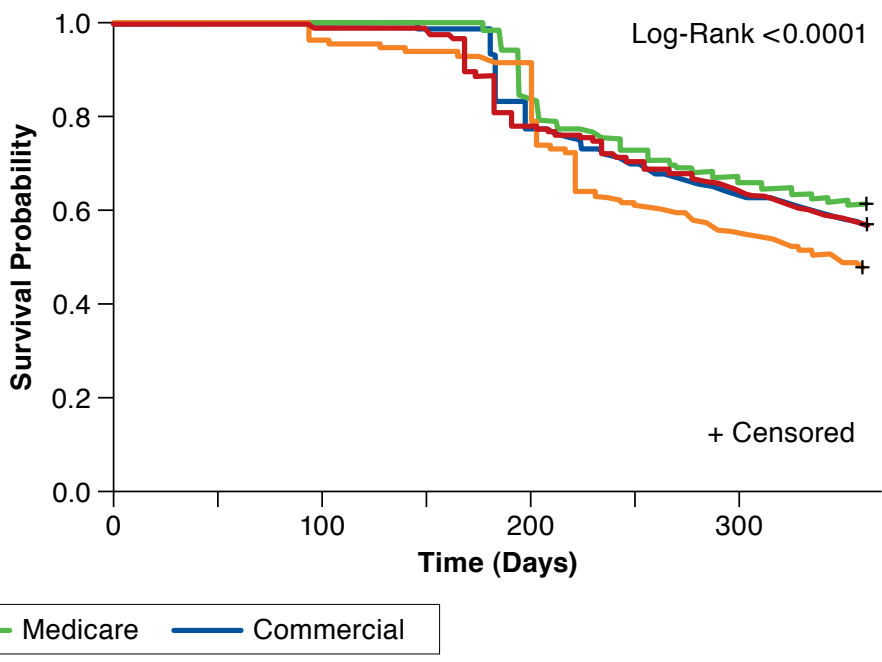

vial/syringe users were significantly more likely to discontinue early than pen users $(P<0.001$; data not shown).

New Users. Among new users, persistence rate for pens was overall $63.7 \%(95 \% \mathrm{CI}=63.2-64.3)$ and for vial/syringe was $57.2 \%$ (95\% CI=56.4-58.0); the persistence rate was significantly higher for pen users versus vial/syringe users for all payers (commercially insured: $61.7 \%, 95 \% \mathrm{CI}=61.0-62.4$ vs. 55.2\%, 95\% CI=54.0-56.4; Medicare: $66.1 \%, 95 \% \mathrm{CI}=65.4-$ 66.9 vs. $59.6 \%, 95 \% \mathrm{CI}=58.5-60.6$; Medicaid: $63.1 \%, 95 \%$ $\mathrm{CI}=60.5-65.7$ vs. $56.3 \%, 95 \% \mathrm{CI}=53.8-58.8$; and cash-pay: 59.1\%, 95\% CI $=53.5-64.6$ vs. $43.7 \%, 95 \% C I=38.6-48.8$; all $P<0.0001$; Figure 4). Persistence duration \pm SD for pen users ranged from $309 \pm 72.6$ to $317 \pm 66.6$ days and for vial/ syringe users ranged from $276 \pm 89.5$ to $308 \pm 71.2$ days; mean duration of persistence was significantly higher for pen versus vial/syringe users for all payers (commercially insured: $309 \pm 72.6$ days vs. $300 \pm 76.8$ days; Medicare: $317 \pm 66.6$ days vs. $308 \pm 71.2$ days; Medicaid: $311 \pm 73.8$ days vs. $299 \pm 80.7$ days; and cash-pay: $312 \pm 65.1$ days vs. $276 \pm 89.5$ days; all $P<0.0001$; Figure 4). Kaplan-Meier curves of time to discontinuation showed that vial/syringe users were significantly more likely to discontinue early than pen users $(P<0.0001$; data not shown).

\section{Multivariate Analysis}

For existing users, multiple logistic regressions on 1-year treatment persistence showed that, compared with commercially insured patients, Medicaid and cash-pay patients were significantly less likely to be persistent (Medicaid: odds ratio $[\mathrm{OR}]=0.921,95 \% \mathrm{CI}=0.883-0.961 ; \quad P=0.0001$; cash-pay: $\mathrm{OR}=0.889,95 \% \mathrm{CI}=0.811-0.975 ; \mathrm{P}=0.0124)$, while Medicare patients were marginally more likely to be persistent (OR=1.082, 95\% CI=1.056-1.108; $P<0.0001$; Appendix A, available in online article). Only NPH users were significantly less likely to be persistent than insulin glargine users (NPH: $\mathrm{OR}=0.896,95 \% \mathrm{CI}=0.859-1.934 ; P<0.0001)$. Pen users were significantly more likely to be persistent than vial/syringe users (OR=1.097, 95\% CI = 1.075-1.119; P<0.0001).

Among new users, cash-pay patients were significantly less likely to be persistent compared with commercially insured patients $(\mathrm{OR}=0.829, \quad 95 \% \quad \mathrm{CI}=0.704-0.975 ; \quad P=0.0234)$, whereas Medicare patients were more likely to be persistent ( $\mathrm{OR}=1.234$, 95\% CI =1.175-1.296; $P<0.0001$; Appendix A). Only NPH users were significantly less likely to be persistent than insulin glargine users (NPH: $\mathrm{OR}=0.643,95 \% \mathrm{CI}=0.586$ 0.706; $P<0.0001)$. Pen users were significantly more likely to be persistent than vial/syringe users $(\mathrm{OR}=1.161,95 \% \mathrm{CI}=$ 1.112-1.212; $P<0.0001$ ).

Cox regression on time to discontinuation of index insulin showed that among existing users, Medicaid and cashpay patients were significantly more likely to discontinue their index insulin early than commercially insured patients (Medicaid: hazard ratio $[\mathrm{HR}]=1.066,95 \% \mathrm{CI}=1.031-1.102$, $P=0.0240 ; \quad$ cash-pay: $\quad H R=1.086,95 \% \quad C I=1.011-1.167$, $P=0.0002$ ), whereas Medicare patients were significantly less likely to discontinue their index insulin early (adjusted hazard ratio $[\mathrm{aHR}]=0.915,95 \% \mathrm{CI}=0.897-0.934, \mathrm{P}<0.0001$; Appendix B, available in online article). NPH users were significantly more likely to discontinue their index insulin early than insulin glargine users (NPH: aHR $=1.109,95 \%$ 

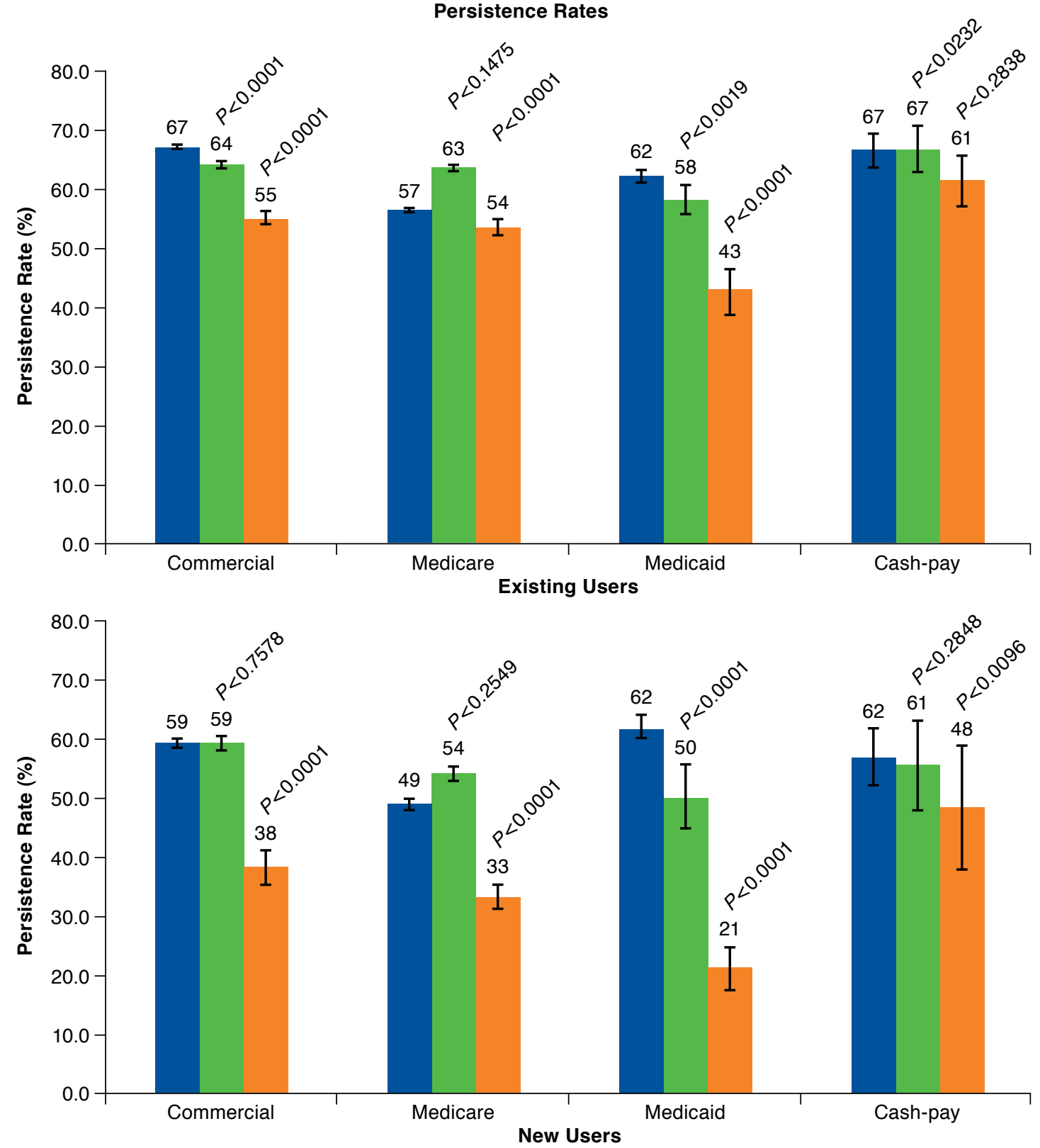

GLA DET $\quad$ NPH

$\mathrm{CI}=1.072-1.147, \mathrm{P}<0.0001)$. Pen users were significantly less likely to discontinue their index insulin early than vial/syringe users ( $\mathrm{aHR}=0.904,95 \% \mathrm{CI}=0.889-0.919, \mathrm{P}<0.0001$ ).

Among new users, Medicare patients were significantly less likely to discontinue their index insulin early than commercially insured patients $(\mathrm{aHR}=0.838,95 \% \mathrm{CI}=0.807-0.869$,
$P<0.0001)$. NPH users were significantly more likely to discontinue their index insulin early than insulin glargine users $(\mathrm{aHR}=1.539,95 \% \mathrm{CI}=1.448-1.635, \mathrm{P}<0.0001$; Appendix B). Pen users were significantly less likely to discontinue their index insulin early than vial/syringe users $(\mathrm{aHR}=0.862,95 \%$ $\mathrm{CI}=0.835-0.890, P<0.0001)$. 


\section{Mean Duration of Persistence}
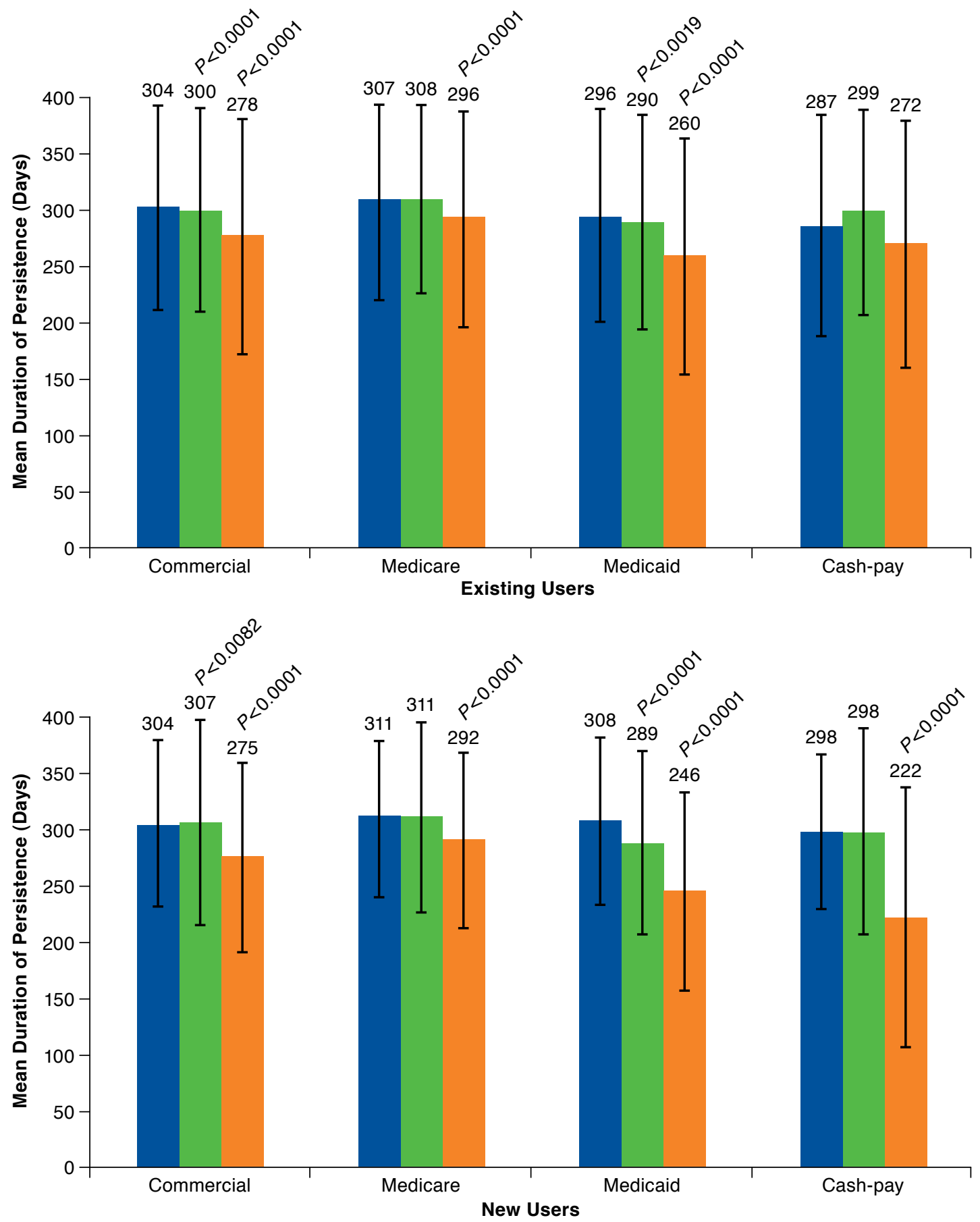

GLA DET $\quad$ NPH

Note: Error bars represent $95 \%$ confidence intervals (persistence rates, previous page) or standard deviations (mean duration of persistence, current page). $P$ values: GLA versus DET or NPH. 

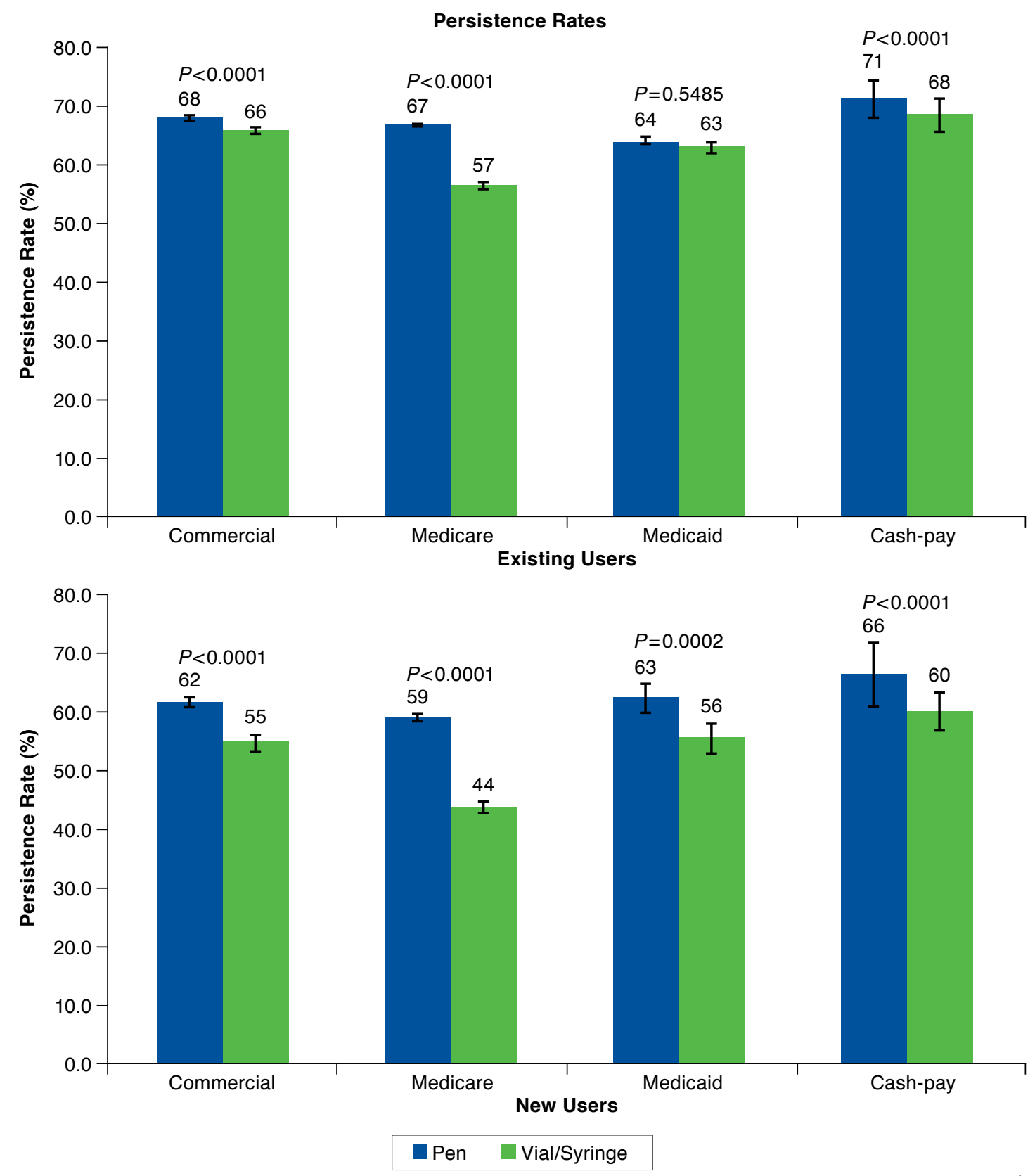

continued on next page

\section{Discussion}

This retrospective, observational study of data from a large national pharmacy database provides the first analysis to benchmark basal insulin treatment persistence among patients with T2D across different payers in the United States, which has not been possible in previous studies using specific insurance claims databases. The findings in this large group of patients highlight significant differences in insulin persistence across different payers, basal insulin types, and devices.

Studies assessing the effects of changing the type of U.S. insurance plan have shown that adherence to insulin therapy is improved after changing to an insurance scheme in which copayments are reduced or even eliminated, suggesting that adherence is improved when the financial burden to the 


\section{Mean Duration of Persistence}
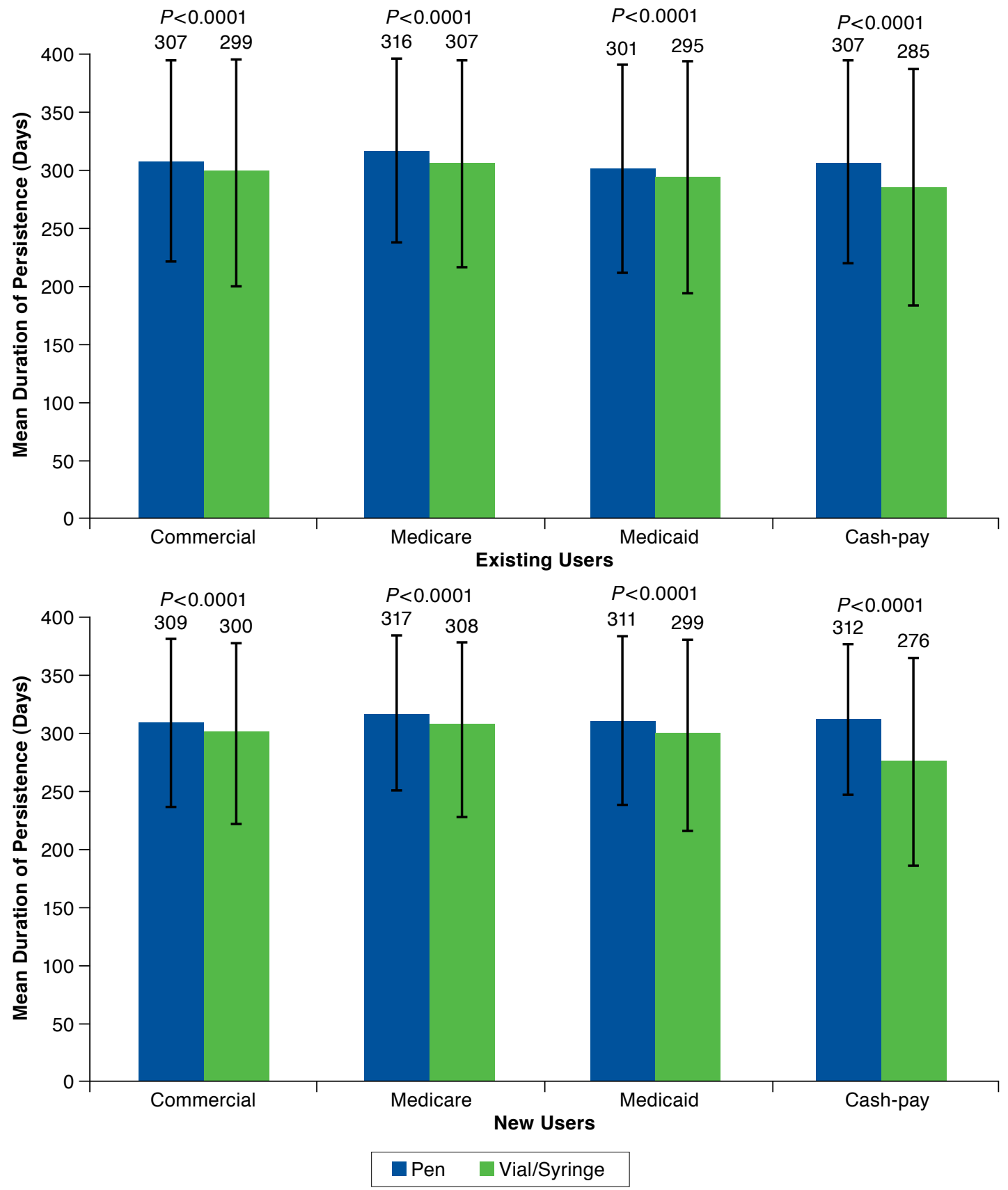

Note: Error bars represent 95\% confidence intervals (persistence rates, previous page) or standard deviations (mean duration of persistence, current page). $P$ values: GLA versus DET or NPH.

DET = insulin detemir; GLA = insulin glargine; NPH=neutral protamine Hagedorn insulin . 
patient is reduced. ${ }^{12}$ In keeping with this, persistence rates in the present study were lower and the duration of persistence shorter among cash-pay patients compared with commercially insured and Medicare patients. In addition, out-of-pocket costs by patients, as measured by the copayment to the index basal insulin, were not a significant predictor of persistence for new users but marginally significant for existing users in that higher copay was associated with lower persistence rate and higher likelihood of earlier discontinuation. This suggested a potential role of insurance benefit design in affecting patients' treatment persistence, particularly among those existing basal insulin users. This information is potentially useful in raising awareness among physicians and targeting efforts to improve persistence among T2D patients in these payer groups. This information is also useful for directing research efforts to explore the specific barriers to improving persistence in these payer groups.

As new basal insulin users are less likely to be on a stable insulin regimen than existing users, and thus change products and be recorded as discontinuing, insulin persistence in the present study was generally higher among existing users (range $=61 \%-70 \%$ ) than new users (range $=51 \%-64 \%$ ). Persistence among new users in the present study is similar to the $65 \%$ 1-year persistence with insulin glargine or insulin detemir in new users reported by Wei et al. (2014). ${ }^{9}$

Insulin persistence in this study was also affected by the insulin delivery device. This finding is in line with numerous other studies showing that persistence is higher with pens compared with vial/syringe delivery., ${ }^{9}-19$ For example, both Miao et al. (2014) $)^{17}$ and Grabner et al. $(2013)^{18}$ reported that persistence with insulin glargine among elderly T2D patients in the United States was significantly higher with a pen device compared with vial/syringe delivery. In an analysis by Xie et al. (2014), ${ }^{15}$ significantly more patients (58\%) were persistent with insulin glargine initiated by pen than with insulin glargine by vial/syringe (51\%) and were persistent for a longer period of time (314 days with pen vs. 299 days with vial/syringe). The present study confirms these findings; in addition, it shows that better persistence with a pen device than a vial is found, irrespective of U.S. payer group or insulin experience, as persistence was higher and the duration of persistence was longer with pens than with vial/syringe administration in all comparisons, although the limitations discussed below should be considered when drawing conclusions.

Many health plans did not cover insulin pen due to its higher cost. In a study by Peyrot and Rubin (2011), ${ }^{20}$ current pen users were more likely than former pen users to have better insurance coverage, with cost reported as the major reason for terminating pen use, indicating that cost and insurance coverage of pens may play an important role in persistence of pen use. All these data support having equal access to insulin pens and vials/syringes across all payer groups. Cost has been reported to be the primary reason for U.S. patients having problems accessing health care ${ }^{14}$ and in accessing pens in particular ${ }^{20}$; thus, addressing economic barriers to the availability of pens in different payer groups may be necessary.

Our data suggest that insulin persistence is also significantly affected by the insulin type. In the present study, persistence was found to be highest with insulin glargine followed by insulin detemir and NPH. This is in line with findings from other studies. For example, in real-world studies of patients with T2D, insulin initiation using insulin glargine was associated with significantly higher persistence compared with insulin detemir $^{9,10}$ and NPH. ${ }^{2,10}$ This may result from the higher efficacy of insulin glargine compared with insulin detemir or NPH and the need for a single dose of insulin glargine compared with often twice-daily dosing of insulin detemir or $\mathrm{NPH}^{2,9}$

Understanding that insulin persistence differs according to payers, basal insulin types, and devices may be useful in developing targeted approaches to improve T2D patient persistence with insulin treatment for better glycemic control. Changes to the U.S. health care system due to implementation of the 2010 Patient Protection and Affordable Care Act that seek to address some gaps in the U.S. private-public insurance system eligibility ${ }^{11}$ make this study particularly relevant and timely, as it provides a historic benchmark to facilitate future review given changes in the insured populations.

\section{Limitations}

As with all retrospective studies, this study may be subject to selection bias and confounding, and no causality relationship can be established. In addition, the analyses were based on data from a single pharmacy database. Without medical claims data, we identified T2D patients by including those who had $\geq 1$ concomitant OAD at baseline; T2D patients who were treated exclusively with insulins at baseline were excluded from the analysis. Although the multivariate analysis controlled for baseline antihypertension and baseline antihyperlipidemia medication usage, this may not be sufficient to control for comorbidities. Therefore, this study may not be representative of other populations or generalizable to all patients with T2D.

Persistence with therapy was estimated using pharmacy claims data that reflect prescriptions filled by the patients; however, medications may not have been taken or consumed as prescribed. The open architecture of the database means that data may not have been complete for patients who filled the studied prescription drugs outside the Walgreens pharmacy system, and actual persistence rates might have been higher reported. The requirement of $\geq 1$ filled prescription in each of the 4 quarters before and after the index basal insulin prescription was put in place as a proxy for eligibility and may have introduced bias by selecting patients more likely to be 
persistent, biasing the data toward higher persistence; actual real-world persistence rates may be lower than reported. New basal insulin analogues such as insulin glargine U300 and insulin degludec were not marketed at the time of the study and therefore were not included in the analysis.

\section{Conclusions}

This large-scale study provides a first look into benchmarking for basal insulin treatment persistence among patients with T2D across different payer segments in the United States. Our findings indicate that insulin persistence patterns are significantly different across different payers, basal insulin types, and devices. This information may be useful in developing targeted approaches to improve T2D patient persistence with insulin treatment for better glycemic control. Additional investigations using the Walgreens database, including additional information (e.g., patient comorbidities, out-of-pocket costs, formulary changes) and more robust statistical analyses such bootstrapping, may be able to address many of the limitations of this study.

\section{Authors}

WENHUI WEI, PhD, MS, MBA, and SOHINI GANGULI, PharmD, Sanofi U.S., Bridgewater, New Jersey. JENNY JIANG, MS; YOUBEI LOU, PhD; and MARK S. MATUSIK, PharmD, Walgreen Co., Deerfield, Illinois.

AUTHOR CORRESPONDENCE: Mark S. Matusik, PharmD, Walgreen Co., 1415 Lake Cook Rd., MS L444, Deerfield, IL 60015. Tel.:847.964.6684; E-mail:mark.matusik@walgreens.com.

\section{DISCLOSURES}

This study was funded by Sanofi U.S. through a grant provided to Walgreens for research services. Matusik, Jiang, and Lou are employed by Walgreen Co. Wei and Ganguli were employed by Sanofi U.S. at the time of this study.

Study concept and design were contributed by Wei, Ganguli, and Matusik, with assistance from Lou. Jiang took the lead in data collection, along with Lou, and data interpretation was performed by Wei, Lou, and Jiang, along with Ganguli and Matusik. The manuscript was written by Wei and Jiang, along with Ganguli and Matusik, and revised by Wei and Ganguli, along with the other authors.

\section{ACKNOWLEDGMENTS}

The authors received writing and editorial support in the preparation of this manuscript provided by Pim Dekker, PhD, of Excerpta Medica, funded by Sanofi U.S.

\section{REFERENCES}

1. Inzucchi SE, Bergenstal RM, Buse JB, et al. Management of hyperglycemia in type 2 diabetes: a patient-centered approach: position statement of the American Diabetes Association (ADA) and the European Association for the Study of Diabetes (EASD). Diabetes Care. 2012;35(6):1364-79.
2. Wang L, Wei W, Miao R, Xie L, Baser O. Real-world outcomes of U.S. employees with type 2 diabetes mellitus treated with insulin glargine or neutral protamine Hagedorn insulin: a comparative retrospective database study. BMJ Open. 2013;3(4):e002348. Available at: http://bmjopen.bmj.com/ content/3/4/e002348.long. Accessed January 18, 2017.

3. Rosenstock J, Dailey G, Massi-Benedetti M, Fritsche A, Lin Z, Salzman A. Reduced hypoglycemia risk with insulin glargine: a meta-analysis comparing insulin glargine with human NPH insulin in type 2 diabetes. Diabetes Care. 2005;28(4):950-55.

4. Home P, Kurtzhals P. Insulin detemir: from concept to clinical experience. Expert Opin Pharmacother. 2006;7(3):325-43.

5. Ng CJ, Lai PS, Lee YK, Azmi SA, Teo CH. Barriers and facilitators to starting insulin in patients with type 2 diabetes: a systematic review. Int $J$ Clin Pract. 2015;69(10):1050-70.

6. Cramer JA, Roy A, Burrell A, et al. Medication compliance and persistence: terminology and definitions. Value Health. 2008;11:44-47.

7. Asche C, LaFleur J, Conner C. A review of diabetes treatment adherence and the association with clinical and economic outcomes. Clin Ther. 2011;33(1):74-109.

8. Bonafede MM, Kalsekar A, Pawaskar M, et al. Insulin use and persistence in patients with type 2 diabetes adding mealtime insulin to a basal regimen: a retrospective database analysis. BMC Endocr Disord. 2011;11:3. Available at: http://www.biomedcentral.com/1472-6823/11/3. Accessed January 18, 2017. 9. Wei W, Pan C, Xie L, Baser O. Real-world insulin treatment persistence among patients with type 2 diabetes. Endocr Pract. 2014;20(1):52-61.

10. Pscherer S, Chou E, Dippel FW, Rathmann W, Kostev K. Treatment persistence after initiating basal insulin in type 2 diabetes patients: a primary care database analysis. Prim Care Diabetes. 2015;9(5):377-84.

11. Davis KL, Tangirala M, Meyers JL, Wei W. Real-world comparative outcomes of U.S. type 2 diabetes patients initiating analog basal insulin therapy. Curr Med Res Opin. 2013;29(9):1083-91.

12. Davies MJ, Gagliardino JJ, Gray LJ, Khunti K, Mohan V, Hughes R. Real-world factors affecting adherence to insulin therapy in patients with Type 1 or Type 2 diabetes mellitus: a systematic review. Diabet Med. 2013;30(5):512-24.

13. Peyrot M, Barnett AH, Meneghini LF, Schumm-Draeger PM. Insulin adherence behaviours and barriers in the multinational Global Attitudes of Patients and Physicians in Insulin Therapy study. Diabet Med. 2012;29(5):682-89.

14. Department for Professional Employees. The U.S. health care system: an international perspective. Fact sheet 2014. Available at: http://dpeaflcio.org/ wp-content/uploads/US-Health-Care-in-Intl-Perspective-2014.pdf. Accessed January 18, 2017.

15. Xie L, Zhou S, Pinsky BW, Buysman EK, Baser O. Impact of initiating insulin glargine disposable pen versus vial/syringe on real-world glycemic outcomes and persistence among patients with type 2 diabetes mellitus in a large managed care plan: a claims database analysis. Diabetes Technol Ther. 2014;16(9):567-75.

16. Xie L, Zhou S, Wei W, Gill J, Pan C, Baser O. Does pen help? A realworld outcomes study of switching from vial to disposable pen among insulin glargine-treated patients with type 2 diabetes mellitus. Diabetes Technol Ther. 2013;15(3):230-36.

17. Miao R, Wei W, Lin J, Xie L, Baser O. Does device make any difference? A real-world retrospective study of insulin treatment among elderly patients with type 2 diabetes. J Diabetes Sci Technol. 2014;8(1):150-58.

18. Grabner M, Chu J, Raparla S, Quimbo R, Zhou S, Conoshenti J. Clinical and economic outcomes among patients with diabetes mellitus initiating insulin glargine pen versus vial. Postgrad Med. 2013;125(3):204-13.

19. Buysman E, Conner C, Aagren M, Bouchard J, Liu F. Adherence and persistence to a regimen of basal insulin in a pre-filled pen compared to vial/ syringe in insulin-naive patients with type 2 diabetes. Curr Med Res Opin. 2011;27(9):1709-17.

20. Peyrot M, Rubin RR. Factors associated with persistence and resumption of insulin pen use for patients with type 2 diabetes. Diabetes Technol Ther. 2011;13(1):43-48. 
APPENDIX A Logistic Regression Analysis for Existing Users and New Users

\begin{tabular}{|c|c|c|c|c|c|c|}
\hline & \multicolumn{3}{|c|}{ Existing Users } & \multicolumn{3}{|c|}{ New Users } \\
\hline & OR & $95 \% \mathrm{CI}$ & $P$ Value & OR & $95 \% \mathrm{CI}$ & $P$ Value \\
\hline \multicolumn{7}{|l|}{ Age, years } \\
\hline 18 to $<30$ vs. 65 to $<75$ & 0.617 & $0.566-0.673$ & $<0.0001$ & 0.778 & $0.664-0.911$ & 0.0019 \\
\hline 30 to $<50$ vs. 65 to $<75$ & 0.691 & $0.669-0.714$ & $<0.0001$ & 0.864 & $0.810-0.921$ & $<0.0001$ \\
\hline 50 to $<65$ vs. 65 to $<75$ & 0.897 & $0.874-0.921$ & $<0.0001$ & 0.970 & $0.918-1.024$ & 0.2687 \\
\hline$\geq 75$ vs. 65 to $<75$ & 0.829 & $0.805-0.854$ & $<0.0001$ & 0.856 & $0.804-0.912$ & $<0.0001$ \\
\hline Gender (female vs. male) & 0.917 & $0.900-0.934$ & $<0.0001$ & 0.964 & $0.928-1.002$ & 0.0647 \\
\hline \multicolumn{7}{|l|}{ Region } \\
\hline Midwest vs. Northeast & 1.072 & $1.037-1.109$ & $<0.0001$ & 1.093 & $1.020-1.171$ & 0.0115 \\
\hline South vs. Northeast & 0.879 & 0.851-0.908 & $<0.0001$ & 0.948 & $0.887-1.012$ & 0.1098 \\
\hline West vs. Northeast & 0.936 & $0.903-0.970$ & 0.0003 & 1.039 & $0.965-1.118$ & 0.3135 \\
\hline \multicolumn{7}{|l|}{ Insulin } \\
\hline DET vs. GLA & 0.986 & $0.963-1.010$ & 0.2631 & 1.010 & $0.965-1.057$ & 0.6622 \\
\hline NPH vs. GLA & 0.896 & $0.859-0.934$ & $<0.0001$ & 0.643 & $0.586-0.706$ & $<0.0001$ \\
\hline Pen vs. vial & 1.097 & $1.075-1.119$ & $<0.0001$ & 1.161 & $1.112-1.212$ & $<0.0001$ \\
\hline \multicolumn{7}{|c|}{ Concomitant medication (yes vs. no) } \\
\hline Metformin & 1.136 & $1.114-1.158$ & $<0.0001$ & 1.195 & $1.148-1.244$ & $<0.0001$ \\
\hline Sulfonylureas & 0.919 & $0.900-0.939$ & $<0.0001$ & 1.235 & $1.185-1.286$ & $<0.0001$ \\
\hline DPP-4 inhibitors & 1.039 & $1.011-1.068$ & 0.0054 & 1.402 & $1.335-1.472$ & $<0.0001$ \\
\hline a-glucosidase inhibitors & 0.916 & $0.799-1.050$ & 0.2083 & 1.447 & $1.129-1.855$ & 0.0035 \\
\hline Meglitinides & 1.020 & $0.946-1.099$ & 0.6081 & 1.148 & $0.995-1.324$ & 0.0584 \\
\hline GLP-1 receptor antagonists & 1.204 & $1.159-1.250$ & $<0.0001$ & 1.561 & $1.448-1.682$ & $<0.0001$ \\
\hline Thiazolidinediones & 1.224 & $1.151-1.300$ & $<0.0001$ & 1.113 & $0.941-1.315$ & 0.2105 \\
\hline Rapid-acting/regular insulin & 1.170 & $1.147-1.193$ & $<0.0001$ & 1.035 & 0.990-1.083 & 0.1263 \\
\hline \multicolumn{7}{|l|}{ Comorbidities (yes vs. no) } \\
\hline Hypertension & 1.109 & $1.08-1.139$ & $<0.0001$ & 1.085 & $1.033-1.140$ & 0.0011 \\
\hline Hyperlipidemia & 1.238 & $1.213-1.264$ & $<0.0001$ & 1.211 & $1.163-1.262$ & $<0.0001$ \\
\hline \multicolumn{7}{|l|}{ Insurance type } \\
\hline Cash-pay vs. commercial & 0.889 & $0.811-0.975$ & 0.0124 & 0.829 & $0.704-0.975$ & 0.0234 \\
\hline Medicaid vs. commercial & 0.921 & $0.883-0.961$ & 0.0001 & 1.080 & $0.988-1.182$ & 0.0915 \\
\hline Medicare vs. commercial & 1.082 & $1.056-1.108$ & $<0.0001$ & 1.234 & $1.175-1.296$ & $<0.0001$ \\
\hline \multicolumn{7}{|c|}{ Copayment for index basal insulin prescription } \\
\hline Copay $>\$ 0$ but $\leq \$ 5$ vs. $\$ 0$ & 1.016 & $0.983-1.051$ & 0.3465 & 0.939 & $0.875-1.008$ & 0.0838 \\
\hline Copay $>\$ 5$ but $\leq \$ 25$ vs. $\$ 0$ & 1.088 & $1.054-1.124$ & $<0.0001$ & 0.899 & $0.925-1.060$ & 0.7773 \\
\hline Copay $>\$ 25$ but $\leq \$ 50$ vs. $\$ 0$ & 1.100 & $1.066-1.135$ & $<0.0001$ & 0.990 & $0.842-0.960$ & 0.0015 \\
\hline Copay $>\$ 50$ vs. $\$ 0$ & 0.972 & $0.941-1.004$ & 0.0896 & 0.886 & $0.828-0.948$ & 0.0004 \\
\hline
\end{tabular}


APPENDIX B Cox Regression Analysis for Existing Users and New Users

\begin{tabular}{|c|c|c|c|c|c|c|}
\hline & \multicolumn{3}{|c|}{ Existing Users } & \multicolumn{3}{|c|}{ New Users } \\
\hline & aHR & $95 \%$ CI & $P$ Value & aHR & $95 \%$ CI & $P$ Value \\
\hline \multicolumn{7}{|l|}{ Age, years } \\
\hline 18 to $<30$ vs. 65 to $<75$ & 1.475 & $1.381-1.574$ & $<0.0001$ & 1.159 & $1.036-1.296$ & 0.0097 \\
\hline 30 to $<50$ vs. 65 to $<75$ & 1.353 & $1.318-1.389$ & $<0.0001$ & 1.104 & $1.050-1.160$ & $<0.0001$ \\
\hline 50 to $<65$ vs. 65 to $<75$ & 1.096 & $1.072-1.120$ & $<0.0001$ & 1.013 & $0.970-1.057$ & 0.5623 \\
\hline$\geq 75$ vs. 65 to $<75$ & 1.172 & $1.144-1.201$ & $<0.0001$ & 1.136 & $1.082-1.194$ & $<0.0001$ \\
\hline Gender (female vs. male) & 1.078 & $1.062-1.095$ & $<0.0001$ & 1.023 & 0.993-1.054 & 0.1262 \\
\hline \multicolumn{7}{|l|}{ Region } \\
\hline Midwest vs. Northeast & 0.945 & $0.919-0.971$ & $<0.0001$ & 0.938 & $0.889-0.990$ & 0.0192 \\
\hline South vs. Northeast & 1.111 & 1.081-1.141 & $<0.0001$ & 1.041 & 0.990-1.096 & 0.1191 \\
\hline West vs. Northeast & 1.056 & $1.026-1.088$ & 0.0003 & 0.968 & $0.914-1.025$ & 0.2697 \\
\hline \multicolumn{7}{|l|}{ Insulin } \\
\hline DET vs. GLA & 1.010 & $0.991-1.030$ & 0.2987 & 0.958 & $0.924-0.992$ & 0.0169 \\
\hline NPH vs. GLA & 1.109 & $1.072-1.147$ & $<0.0001$ & 1.382 & $1.297-1.473$ & $<0.0001$ \\
\hline Pen vs. vial & 0.904 & $0.889-0.919$ & $<0.0001$ & 0.878 & $0.849-0.907$ & $<0.0001$ \\
\hline \multicolumn{7}{|c|}{ Concomitant medication (yes vs. no) } \\
\hline Metformin & 0.899 & $0.885-0.914$ & $<0.0001$ & 0.865 & $0.839-0.893$ & $<0.0001$ \\
\hline Sulfonylureas & 1.077 & $1.059-1.096$ & $<0.0001$ & 0.854 & $0.827-0.882$ & $<0.0001$ \\
\hline DPP-4 inhibitors & 0.970 & $0.948-0.992$ & 0.0077 & 0.759 & $0.729-0.790$ & $<0.0001$ \\
\hline a-glucosidase inhibitors & 1.084 & $0.970-1.211$ & 0.1544 & 0.735 & $0.595-0.908$ & 0.0043 \\
\hline Meglitinides & 0.989 & $0.929-1.053$ & 0.7293 & 0.896 & $0.798-1.005$ & 0.0611 \\
\hline GLP-1 receptor antagonists & 0.849 & $0.823-0.877$ & $<0.0001$ & 0.693 & $0.651-0.737$ & $<0.0001$ \\
\hline Thiazolidinediones & 0.843 & $0.801-0.888$ & $<0.0001$ & 0.918 & 0.801-1.052 & 0.2178 \\
\hline Rapid-acting/regular insulin & 0.872 & $0.858-0.887$ & $<0.0001$ & 0.961 & $0.928-0.994$ & 0.0209 \\
\hline \multicolumn{7}{|l|}{ Comorbidities (yes vs. no) } \\
\hline Hypertension & 0.917 & $0.898-0.937$ & $<0.0001$ & 0.941 & $0.907-0.977$ & 0.0013 \\
\hline Hyperlipidemia & 0.836 & $0.823-0.851$ & $<0.0001$ & 0.861 & $0.834-0.888$ & $<0.0001$ \\
\hline \multicolumn{7}{|l|}{ Insurance type } \\
\hline Cash-pay vs. commercial & 1.086 & $1.011-1.167$ & 0.0240 & 1.113 & $0.992-1.249$ & 0.0690 \\
\hline Medicaid vs. commercial & 1.066 & 1.031-1.102 & 0.0002 & 0.967 & $0.903-1.036$ & 0.3381 \\
\hline Medicare vs. commercial & 0.915 & $0.897-0.934$ & $<0.0001$ & 0.811 & $0.780-0.842$ & $<0.0001$ \\
\hline \multicolumn{7}{|c|}{ Copayment for index basal insulin prescription } \\
\hline Copay $>\$ 0$ but $\leq \$ 5$ vs. $\$ 0$ & 0.992 & $0.965-1.019$ & 0.5413 & 1.045 & $0.989-1.105$ & 0.1134 \\
\hline Copay $>\$ 5$ but $\leq \$ 25$ vs. $\$ 0$ & 0.934 & $0.910-0.959$ & $<0.0001$ & 1.010 & $0.958-1.065$ & 0.0006 \\
\hline Copay $>\$ 25$ but $\leq \$ 50$ vs. $\$ 0$ & 0.925 & $0.901-0.949$ & $<0.0001$ & 1.092 & $1.038-1.148$ & 0.7073 \\
\hline Copay $>\$ 50$ vs. $\$ 0$ & 1.029 & $1.002-1.056$ & 0.0373 & 1.095 & $1.039-1.154$ & 0.0007 \\
\hline
\end{tabular}

